\title{
Sachregister zu Band 62
}

Die fett gedruckten Zahlen bezeichnen Eigenarbeiten. Bb. = Buchbesprechung. A.

Ablatio retinae, erfolgreichePunk-tion einer - 102 .

Skleraresektion wegen -410 .

und Betriebsunfall 357. Adaptation, Weber-Fechnersches

Gesetz und - 339. Aderhaut s. Chorioidea. Adrenalin, Behandlung des Früh-

jahrskatarrhs mit -86 .

- $\quad$ Behandlung der Iritiden mitDrucksteigerung mit -87 .

Akkommodation, Schädlichkeit der -- 193.

- Wirkung der Ermüdung auf die -

309 .

Akkommodationsbreite bei Al-

koholikern 288. Akkommodationsmuskulatur,

Pharmakologie der-325. Alkoholiker, Akkommodationsbreite bei-288. Alkoholinjektionen,

die Kera-titis neuroparalytica nach - ins Ganglion Gasseri 1. Alkoholintoxikation, Verände-

rungen der Uvea bei chronischer

- 90 .

Aneurysma arteriovenosum der

Vena ophthalmica sup. nach Schä-

delbasisbruch 195. Angiocavernom der Orbita 120. Angiolymphe, Wirkung der - bei

tuberkulösen Augenerkrankun-

gen 192. Aniridie, beiderseitige kongenitale

- 184.Arachnodaktylie, Augenbefund

bei - 345-

Argentum nitricum-Lösung, Hornhautverätzung durch Ein-tropfen einer ioproz. - 126.

Arteria centralis retinae, Behandlung des Verschlusses der - oder eines ihrer Äste 257.

Assoziierte Bewegungen, Stö-rungen der -316 .

Astigmatismus, Aufzeichnung des

- 352-

- $\quad$ Bedeutung der Skiaskopie beim

$-\quad 3^{\circ} 9$.

- Bestimmung des - mit Hilfe der

Farbenzerstreuung 340. -- Korrektion des - 108, 209.

- $\quad$ und Myopie 309.

Augapfel s. Bulbus.

Augendruck s. Druck.

Augenhintergrund, Befund am - bei tuberöser Hirnsklerose 73.

Augenhintergrund, perlschnur-artige Pigmentstreifen im - 131.

- Veränderungen des - während der Behandlung mit Neosalvar-san 87. 
Augenlider s. Lider.

Augenmuskeln, funktionelle Bedeutung der Sechszahl der -318 .

Augensalben, chemische Reaktion und Abgabefähigkeit von - 337 .

Augenverletzungen 311, 353.

Automobilauspuff gas, Kohlen-oxydvergiftung durch - 126 .

Avitaminosenund Hemeralopiegv.

B.

Bacillus fusiformis bei akuter

Conjunctivitis 370. Bakterioskopie des Bindehaut-

sackes 353. Basedow-Exophthalmus, beiderseitige Erblindung infolge von

114. Bindehaut s. Conjunctiva. Biologische Arbeitsmethoden,

Handbuch der - 360 (Bb.). Bläschenbildung bei Hornhaut-

erkrankungen 336. Blepharochalasis mit beiderseiti-

ger Luxation der Tränendrüse 118 Blinde, Registration der - in der

Ukraine 117. Blutdruckinden intraokularen Ge-

fäßen 319. Blutreaktion bei Glaukomkranken

119 Blutversorgung der Korneoskle-

ralgrenze 345. Brillengläser, Vergrößerung durch

-310. Broncestaubbetriebe, gewerbe-

hygienische und klinisch-röntgeno-

logische Untersuchungen in -125. Bubonenpest, Augenveränderun-

gen bei -88 .

C.

Chalcosis corneae, atypische --bei fehlerhafter Kupferstiftbe-handlung 180.

Sachregister. 417

Chiasma, vergleichend-morpholo-

gische Ausgestaltung der ge-

gend 327. Chinin-Arsenkur bei Netzhaut-

blutungen nach Malaria 86. Chininintoxikation 190. Chlorom 373. Cholesterinkristalleauf

derNetz-

haut 98. Chorioidea, pathologische Ana-

tomie und Therapie des Solitär-

tuberkels der -354 .

-abhebung, Entstehung der bei-derseitigen spontanen hinteren - 186.

-karzinom, metastatisches 188.

-sarkom, Bedeutung des Kam-merwassers bei - 334.

- Zwei - -sarkome in einem Augapfel 349.

Chronometrage in den Augenab-

teilungen in Kiew 353. Conjunctiva bulbi, Röntgenbe-

handlung bei neoplastischer Tu-

berkulose der -93 .

- $\quad$-plastik 199.

- $\quad$-sack, Bakterioskopie des - 353.Conjunctivitis hyperplastica lym-

pho-adenoides diffusa 341.

- $\quad$ Spirochäten und Bacillus fusi-m formis bei akuter -370 .

Cornea, atypische Chalcosis der - bei fehlerhafter Kupferstift-behandlung 180. 
Bild der Uveitis nach Verbren-nung der - 196.

-epithelveränderungen bei einem Fall von myotoner Dystrophie 44.

Erkrankungen der - 335, 336.

-geschwöre, Radiumbehandlung fistelnder 41.

Klinik und Pathologie seltener Erkrankungen der - 21 .

-oberfläche, optische Darstel-lung der - 319.

oberflächliche Splitterverletzun-gen der-311.

reflexbild, Photometrie des -

334- '

- $\quad$-skleralzyste 184.

Speicherzellen in der -- 349. staphylome, angeborene 325.

und Lidspalte 145.

Corpus vitreum, Parasiten im -381 .

- $\quad$ Veränderungen des -82 .

D.

Daueranästhesie, bei schmerzhaf-ten Augenaffektionen 98.

Degenerative Veränderungen des Auges, Pathogenese der - $34^{\circ}$.

Diphtherieheilserum, Heteropro-teintherapie mit - bei krupöser Pneumokokkeninfektion der

Bin-dehaut 87.

Doppelbilder, Aufzeichnung der

- 352. Doppelbilderuntersuchungen,

Lampe für - 116. Drechslerarbeit, Schädigung der

Augen durch - 127. Druck, intraokulärer, Einíluß

endokriner Drüsen auf den -88 .

Einfluß von Lösungen verschie-dener Konzentration auf den -82 .

des Kaninchenauges, Wirkung einiger Arzneimittel auf den - 221.

- $\quad$ und die äußeren Bulbushüllen 31.9.Druckbeeinflussung bei Sekun-

därglaukom durch Salvarsan 197. Druckmessung, intrakranielle -

mit Hilfe der Netzhautvenenpuls-

beobachtung 323. Drucksenkung bei Keratitis her-

petica des Kaninchens 227. Dunkeladaptation, Ablauf der

338. Dunkelnystagmus, Verhalten des

- nach zentraler Vestibularaus-

schaltung 348. Dyskeratosis corneae conge-

nita 21. Dystrophia adiposa corneae 21. Dystrophie, Hornhautepithelver-

änderungen bei einem Fall von

myotoner -44 .

E. Eckzähne, Erhebung der - des

Unterkiefers bei Heredosyphilis

202. Ectopia lentis et pupillae, dop-

pelseitige - als Begleiterschei-

nungeiner Konstitutionsanomalie

394. Eigenblutinjektionen 412. Einschlußinfektion der Binde-

haut beim Erwachsenen 187. Elastometrie des Auges 321. Elephantiasis des Oberlides, Zu-

sammenhang der einseitigen -

mit Erweiterung der Sella turcica 
344-Emetin 313. Endokrine Drüsen, Einfluß der auf die Pupille und den intraokulären Druck 88. Endophthalmitis septica, endogene - nach Staroperation 114. Endotheliom des Keilbeins 192. Enukleation, Fetteinpflanzung nach -411 .

$28^{*}$

$4 \mathrm{i} 8$

Sachregister.

Epitheliom, multiple 411. Epitarsus, zur Genese des - 63. Ermüdung, Wirkung der - auf die Akkommodation 309. Erysipel, induratives Lidödem bei

chronischem rezidivierenden -

192. Euphorbia Helioscopa, Binde-

haut- und Hornhautentzündung

durch Saft der-358. Evulsio nervi optici 81. Explantationsversuche mit Au-

gengeweben 325 .

$\Gamma$.

Farbensehen, Theorie des -329 .

Farbensinn 91.

- Übergangsformen zwischen nor-

malem - und anormaler Trichro-

masie 329. Farbenzerstreuung, Bestimmung

des Astigmatismus mit Hilfe der

- 34ㅇ-Farbphotographien des Auges,

stereoskopische - 347. Fett am Auge bei Keratomalazie

und Herpes 335. Fetteinpflanzung nach Enuklea-

tion 411. Fettröpfchen in der Vorderkam-

mer nach perforierender Ver-

letzung 127. Fluorwasserstoffsäure, schwere

Verätzung durch - 125. Flüssigkeiten des Auges, oxydie-

rende und reduzierende Wirkung

der -312 . Flüssigkeitswechsel in der vor-

deren Kammer 310. Fremdkörper 91, 92, 106, 127,

188, 345-Frühj ahrskatarrh, Behandlung

des - mit Adrenalin 86.

G.

Ganglion Gasseri, die Keratitis

neuroparalytica nach Alkoholin-

jektionen in das - 1. Gesichtsfeldaufnahmen, Verein-

heitlichung der. - 97, 330. Glaskörper s. Corpus vitreum. Glasleistenbildung an der Horn-

hauthinterfläche bei Keratitis

parenchymatosa 230. Glasmacherstar 333, 356. Glassplitter im Auge 188. Glaukom 82, 83, 320, $321,322$.

- $\quad$ Die aktuelle Blutreaktion bei

kranken 119.

- $\quad$ Differentialdiagnose zwischen Gl.simplex und Sehnervenatrophie104. 
Glaukom, Druckbeeinflussung beiSekundär durch Salvarsan 197.

einfaches - als Unfallfolge nicht anerkannt 122.

Messung der Vorderkammertiefe beim - 334.

Gliom im Linsenkern mit ungewöhn

lichen Augensymptomen 246. Goldbehandlung der Lepra 354.

$\mathrm{H}$.

Hämangiom der Orbita mit Venen-knäuel an der Papule 199.

Hemeralopie und Avitaminosen 90.

Hemianopsie, Differentialdiagnose der infra- und supranukleären -

324-Herpes, Fettvorkommen am Auge

bei - 335. Herpes corneae, Dauer eines 76 .

- experimenteller 314. HeteroproteintherapiemitDiph-

therieheilserum bei krupöserPneu-

mokokkeninfektion der Binde-

haut 87. Hirnsklerose, Augenhintergrund-

befund bei tuberöser - 73. Hyperthyreoidismus, Verlauf der

chirurgischen Verletzungen des

Auges bei experimentellem - 91. Hypophysengeschwülste 194. Hypophysensymptome bei den

Terrakottafiguren in Kleinasien

90. Hyp otonie 321 .

I.

Inf ektionsübertragung von Bul-

bus zu Bulbus 335. Insulin und augenärztliche Chirur-

gie beim Diabetiker 87. Intracisternale Injektionen bei

luetischer Optikusatrophie 414. Intraokulare Flüssigkeit, Her-

stellung der -218 .

- $\quad$ Refraktometrie und Eiweißbe-stimmung der - 340.

Iridenkleisisantiglaucomatosa

322. Iridocyclitis tuberculosa 113. Iris, Aufzeichnung der Farbe der -

352.

Entstehung der Pigmentation auf der - 185.

Lymphwege der -318 .

-schw und bei kongenitaler Tabes 348 .

- $\quad$ tuberkulöse Geschwulst der - 95.Irisverfettung, Entstehung der -

344-Iritis mit Drucksteigerung, Behandlung der - mit Adrenalin 87.

- $\quad$ Uricämie bei -89 .

Sachregister.

419

$\mathrm{J}$.

Jodtinkturbei trachomatöser Con-junctivitis 87 .

K.

Kalkverätzung mit Milchtherapie

behandelt. 87. Rammer, Entstehung der Verfet-

tung der vorderen -344 .

- $\quad$ Messung der Tiefe der - 334.Kammerwasser, Bedeutung des - 
bei Aderhautsarkom 334.

- $\quad$ Einfluß von Lösungen verschie-dener Konzentration auf denStickstoffgehalt des -82 . Katarakt 332, 333, 351.

- $\quad$ konservative Behandlung der -

i93 - Kataraktoperation 331, 332, 356, 411, 412, 415.

endogene Endophthalmitis sep-tica nach - 114.

tonometrische Messungen nach - 94 .

Keilbein, Endotheliom des - 192. Keratitis herpetica des Kaninchens, Drucksenkung bei - 227.

neuroparalytica, die Grundlagen der -1 .

- beiderseitige -, kombiniert mit basalen Lähmungen und psy-chischem Defekt 113.

- durch Gewebsschädigung 93.

parenchymatosa, Behandlung der -411 .

- Behandlung der - - - e lue hereditaria 168.

- Einfluß der Salvarsanbehand-lung der Elterngeneration auf die Abnahme der - 121.

- Glasleistenbildung an der Hornhauthinterfläche bei - 230 .

- und Lues congenita bei zwei Generationen 100.

- Vakzine- - 343.Keratohypopyon, Behandlung

des -86 . Keratomalazie, Fettvorkommen

am Auge bei - 335. Koch-Weekssche Infektion 337. Kohlenoxydvergiftung durch

Automobilpuffgas 126. Kongruenzapparat 350. Konstitutionelle Pathologie in

der Augenheilkunde 91. Kontusionskatarakt 351. Kraftfahreranwärter, die Zeug-

nisse der - 359. Kupfer im Auge 93. Kupfersplitter Extraktion eines

doppelperforierenden - aus der

Orbita unter Erhaltung des Auges

127.

Kupf erstiftbehandlung, atypi-sche Chalcosis corneae bei fehler-hafter - 180 .

L.

Lampe für Doppelbilderuntersu -chungen 116.

Leishmaniosis der Lidhaut 344.

Lentiglobus 351.

Lepra, Goldbehandlung der -354

Leprome, Überimpfung von Frag-menten und Emulsionen von - in die Vorderkammer und Hornhaut

.315-Lid, blaue Verfärbung des unteren

- $\quad$ nach Sondierung des Tränen-kanals 96.

- $\quad$ Instrument zum Umstülpen des

- 116.

-bindehautkarzinom, bei einem zwölfjährigen Knaben 261.

-ödem, induratives bei chroni-schem rezidivierenden Erysipel 192.

-plastik 199.

-spaltenanomalie 93.

- $\quad$ und Hornhaut 145.Limbusknötchen bei Varizellen

185. Linsenatmung 333.

- professionelle Schädigungen der

- $\quad$ bei Glasmachern 333, 356.Linsenkern, Gliom im - mit un-

gewöhnlichen Augensymptomen 246. Linsenluxation, experimentelle 316. 
- $\quad$ familiäre 162.Linsenreste, Bedeutung der - für

die postoperativen Entzündun-gen 342. Linsentrübungen, symmetrische

$35 \mathrm{i} \cdot$ Lochbildung in der Makula nach

Sprengschußverletzung 200. Lokalanästhesie in gerichtsärzt-

licher Bedeutung 126. Lokalisationstherapie 341. Lumbalanästhesie, Augenerschei-

nungen bei - 90. -- und Lumbalpunktion, Beweg-

lichkeitsstörungen des Auges nach

- $\quad$ 317· Lymphwege der Iris 318.

M.

Makula, eigenartige Veränderung der - 399.

- $\quad$ Lochbildung in der nach-Sprengschußverletzung 200.

Malaria, Chinin-Arsenkur bei Netz-hautblutungen nach - 86 .

420

Sachregister.

Malariabehandlung der Tabes, besonders der tabischen Optikus-atrophie 155.

Megalokornea 345.

Meningismus und Meningo-En-zephalismus der Augenkranken

323-Melanotische Geschwülste der

Bindehaut, künstliche Entstehung

von - 347. Metallstau b, Schädigung der Horn-

haut durch - 336 . Milchtherapie 85, 87.

- $\quad$ bei Kalkverätzung 87.Mißbildungen, experimentelle Er-

zeugung von Augen - 324. Mitbewegungen 350. Mizuosches Phänomen bei Ogu-

chischer Krankheit 348. Musculusobliquus superior, iso-

lierte Durchreißung des - 282. Musculus rectus internus, post-

traumatische Erkrankung des -

353-Myopie 3o8ff.

- Vererbung der -49.

N. Nachstaroperation 356, 411, 415. Neosalvarsan, Veränderungen des

Augenhintergrundes während der

Behandlung mit -87 . Nervus abducens, Parese des -

399, 4OI· Nervus opticus 415.

-atrophie, Differentialdiagnose der Leberschen familiären - 57.

--, Differentialdiagnose zwischen Glaucoma simplex und - 104.

- intracisternale Injektionen bei luetischer - 414 .

- Malariabehandlung der tabischen -- 155.

Netzhaut s. Retina. Neurologie, ophthalmologische Be-

ziehungen in der -412 . Nystagmus 316, 330.

- $\quad$ Verhalten des Dunkel- - nachzentraler Vestibularausschaltung348.

0 .

Oguchische Krankheit mit Mi-zuoschem Phänomen 348.

Ohrbefunde bei Abduzensparese 401.

Operative Technik 331, 332.

Ophthalmia sympathica 84, 301.

Ophthalmodynamometer, modi-fiziertes Landoltsches 113. 
Ophthalmoplegie, chronische progressive 98.

Ophthalmoskop, elektrisches -

mit Spaltlampe 107. Optochin bei Blennorrhoe des Trä-

nensackes 314. Optophon 97. Orbitalerkrankungen 191. Orbitaltumor 95, 120, 347. Osteoma

osteoblasticum der Or-

bita 95 .

P.

Papilloma conjunctivae bulbi

114. Parasiten im Glaskörper 381. Parotitis epidemica, Skleritis pe-

ricornealis nach -342 . Perimetrie 307ff. Peripupillometer 96. Perprotasin 182. Phlyktäne,

Ätiologie der - 315. Pigmentation, Entstehung der -

auf der Iris 185. Pigmentstreifen, perlschnurartige

im Augenhintergrund 131. Pilokarpineinträuf elung, Vor-

täuschung von Pupillenstörungen

durch - 196. Pilzkonkremente im Tränenröhr-

chen 367. Pneumokokkeninfektion der

Bindehaut, Heteroproteinthera-

pie mit Diphtherieheilserum bei

- 87. Polycythaem ia rubra mit $\mathrm{Au}$.

genhintergrundsveränderungen

353-

Projektionsbilder, stereoskopi-sche - 349-

Pseudosympathisierende Ent-zündung 272.

Pseudotumor der Orbita 347.

Ptosis atonica 118.

Pupillarsaumknötchen, Entstehung der - 338 .

Pupille, Einfluß endokriner Drüsen auf die - 88 .

- $\quad$ Vortäuschung von -Störungendurch Pilokarpineinträufelung196.

Pupillenreflex, Apparat zum Stu-dium der Physiologie und Patho-logie des - 96.

- $\quad$ intrazerebrale Bahn des - 326.Purkinj e-Sansonsche Reflex-

bilder 405 .

R.

Radiumbehandlung fistelnder

Hornhautgeschwüre 41. Recklinghausensche Krankheit,

Augenveränderungen bei - 352. Refraktion 307ft.

Sachregister.

421

Refraktionsbestimmung, ein

neues Instrument zur objektiven

- 78. Refraktionsindex, Einfluß von

Lösungen verschiedener Konzen-

tration auf den -82 . Refraktometer 346. Refraktometrie und Eiweißbe-

stimmung der intraokularen Flüs-

sigkeiten 340. Replantationsmöglichkeiten

des Vertebratenauges 202. Retina-Blutungen, Chinin-Arsen- 
kur bei - nach Malaria 86.

Cholesterinkristalle auf der -98 .

experimentelle Septojoddegene-ration der -339 .

Topographie der Faserverteilung in der -324.

venenpulsbeobachtung, intrakra-nielle Druckmessung mit Hilfe der - 323.

Röntgenbehandlung bei neopla-stischer Tuberkulose der Conjunctiva 93.

Röntgenlokalisation am Aug-apfel 355.

S.

Salvarsan, Druckbeeinflussung bei Sekundärglaukom durch - 197.

- $\quad$ Einfluß der - -behandlung derElterngeneration auf die Ab-nahme der Keratitis

parenchymatosa 121 .

Schädelbasisbruch, Aneurysma arteriovenosum der Vena oph-thalmica sup. nach - 195.

Schneidezähne, Erhebung der •-des Unterkiefers bei Heredosyphi-lis 202.

Schule für Sehschwache 198.

Schutzgläser mit dünnen Metall-schichten 126.

Schwimmbadconjunctivitis, Beziehungen der - zum Trachom

"5.

- $\quad$ eine nicht durch Schwimmbadverursachte - 95 .

Schwimmer, Sehen der - unter Wasser 308.

Sculcosches Mittel bei der Behandlung des Trachoms 85 .

Sehnerv s. Nervus opticus.

Seh $1 / 8$ chärfe, warum ist die bino-kuläre - größer als die mono-kuläre ? 407.

Sehschwache, Einrichtung einer Schule für - 198.

Sella turcica, Zusammenhang der einseitigen Elephantiasis des Ober-lides mit Erweiterung der -344.

Septojoddegeneration, experimentelle - der Netzhaut 339.

Siderosis. der Linse 345. Skiaskopie 309.

- $\quad$ Modell für Erklärung der sk.Strahlenbewegung 340.

Skleraresektion wegen Netzhaut-

ablösung 410. Skleritis, maligne, sog. sulzige 103.

- $\quad$ pericornealis nach Parotitis epi-demica 342.

- $\quad$ Uricämie bei - 89.Spaltlampe 351.

- $\quad$ Verwendung eines astigmatischenStrahlenganges an der - 346.

Spatel, Vorteile des -85 . Speicherzellen in der Hornhaut

349-Spirochäten bei akuter Conjunctivitis 370 .

- $\quad$ bei fötaler Syphilis des Auges 88. Staphylome, angeborene - der

Hornhaut 325. Stauungspapille, doppelseitige -

bei Werlhofscher Krankheit 95. Strabismus 411.

- $\quad$ Behandlung des - 331.Stereokammer zur Aufnahme des

vorderen Bulbus 349. Streifenprobe 340. Sympathikusheterochromie

341 -

Sympathikusreizmittel, Wir-

kung der - 325. Syphilis des Auges 314.

Spirochätenbefund bei fötaler - des Auges 88 .

Wismutbehandlung bei luetischen Augenerkrankungen 86.

Syphiliscongenita, Erhebung der Schneide- und Eckzähne des Un-terkiefers bei-202. 
Tabes, Irisschwund bei kongenitaler

- $34 »$.

- $\quad$ Malariabehandlung der - 155.Teer, völliger Ausguß der Conjunc-

tivalsäcke mit - 359-Tintenstiftverletzungen 359. Tonometrie 83, 94. Trachom 187, 411.

Behandlung des - mit Jodtink-tur 87.

Beziehungen der Schwimmbadconjunctivitis zum - 115 .

chemotherapeutische Versuche beim - 116.

chirurgische Behandlung des -86 .

die degenerativen Stigmata der

- $\quad$-kranken 341.

422 Sachregister.

Trachom, Immunisierungsversuche

beim - 310, 311. -- Wirksamkeit des Sculcoschen Mit-

tels bei der Behandlung des -85 . Tränendrüse, beiderseitige Luxation der - mit

Blepharocholasis

118.

- $\quad$ tuberkulose Entzündung der -114 .

Tränendrüsenentzündung, beiderseitige syphilitische -119 .

Tränenkanal, blaue Verfärbung des Unterlides nach Sondierung des - 96 .

Tränenröhrchen, Pilzkonkremen-te im - 367.

Tränensackblennorrhoe, Opto-chin bei -314 .

Transplantation von Rattenau-ge $\prod 315$.

Trichromasie, Ubergangsformen zwischen anormaler - und nor-malem Farbensinn 329.

Tuberkulose, Röntgenbehandlung bei neoplastischer - der Conjunctiva bulbi 93.

und Zigarrenarbeit 359.

Wirkung der Angiolymphe bei tub. Augenerkrankungen 192.

U.

Ultrarote Strahlen, Schädigun-gen des Auges durch kurzweïlige - 201.

Unfallkunde i2iff., 357.

Unfallneurose und Betriebsunfall 124.

Unterlid, operierter Fall von ab-gerissenem - 116.

Uricämie bei Iritis und Skleritis 89.

Uveitis, Bild der - nach Verbrennung des Gesichts und der Horn-haut 196.

V.

Vakzine-Keratitis 343. Varizellen, Limbusknötchen bei-

185. Velonoskiaskopie Ill, 340. Verbrennung des Gesichts und der

Hornhaut, Bild der Uveitis nach

- 196.

Vererbung in der Augenheilkunde

411. Verkupferung des Auges 174. Versicherungskunde 121 ff., 357. Vestibularausschaltung, Ver-

halten des Dunkelnystagmus nach

- 348.

Viscum album, Wirkung des - auf das Auge des Kaninchens 86. 
Vistosan 85.

Volumen des Kaninchenauges, Wirkung einiger Arzneimittel auf das

221.

W.

Web er-Fechnersches Gesetz

und Adaptation 339. Werlhofsche Krankheit mit dopseitiger Stauungspapille 95. Wismutbehandlung bei luetischen

Augenerkrankungen 86.

$\mathrm{X}$.

Xanthomatosis bulbi 344.

Z.

Zigarrenarbeit und Tuberkulose

359-Zilie in der vorderen Kortikalis 345. Zinkhüttenarbeiter, Untersu -

c. chungen an - 359-Zonulalamelle

Ablösung der - bei

Glasbläsern 334. Zyklopie

Genese der -317 . 